\title{
Article \\ Influence of Oak Chips and Oak Barrel Ageing on Volatile Profile in Chardonnay Wine of Romania
}

\author{
Diana Ionela Stegăruș ${ }^{1,+}$, Anamaria Călugăr ${ }^{2,+} \mathbb{D}$, Corneliu Tanase ${ }^{3, * \mathbb{D}}$, Adriana Muscă ${ }^{3}$,

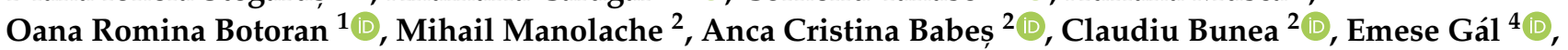 \\ Andrea Bunea ${ }^{5}$ and Teodora Emilia Coldea ${ }^{6, *(\mathbb{D})}$
}

1 National Research and Development Institute for Cryogenics and Isotopic Technologies-ICSI Ramnicu Valcea, 4th Uzinei Street, 240050 Ramnicu Valcea, Romania; diana.stegarus@icsi.ro (D.I.S.); oana.dinca@icsi.ro (O.R.B.)

2 Viticulture and Oenology Department, Faculty of Horticulture, Advanced Horticultural Research Institute of Transylvania, University of Agricultural Sciences and Veterinary Medicine Cluj-Napoca, 3-5 Manastur Street, 400372 Cluj-Napoca, Romania; anamaria.calugar@usamvcluj.ro (A.C.); mihail.manolache@yahoo.com (M.M.); ancababes@usamvcluj.ro (A.C.B.); claudiu.bunea@usamvcluj.ro (C.B.)

3 Department of Pharmaceutical Botany, "George Emil Palade" University of Medicine, Pharmacy, Sciences and Technology of Târgu Mureș, 38 Gheorghe Marinescu Street, Târgu Mureș, 540139 Mureș, Romania; m_adriana_s@yahoo.com

4 Faculty of Chemistry and Chemical Engineering, Babeș-Bolyai University,11 Arany Janos Street, 400028 Cluj-Napoca, Romania; emese@chem.ubbcluj.ro

5 Department of Chemistry and Biochemistry, Faculty of Animal Science and Biotechnology, University of Agricultural Sciences and Veterinary Medicine Cluj-Napoca, 3-5 Manastur Street, 400372 Cluj-Napoca, Romania; andrea.bunea@usamvcluj.ro

check for updates

Citation: Stegăruș, D.I.; Călugăr, A.; Tanase, C.; Muscă, A.; Botoran, O.R.; Manolache, M.; Babeș, A.C.; Bunea,

C.; Gál, E.; Bunea, A.; et al. Influence of Oak Chips and Oak Barrel Ageing on Volatile Profile in Chardonnay Wine of Romania. Appl. Sci. 2021, 11, 3691. https://doi.org/10.3390/ app11083691

Academic Editor: Maria Kanellaki

Received: 23 March 2021

Accepted: 14 April 2021

Published: 19 April 2021

Publisher's Note: MDPI stays neutral with regard to jurisdictional claims in published maps and institutional affiliations.

Copyright: (c) 2021 by the authors. Licensee MDPI, Basel, Switzerland. This article is an open access article distributed under the terms and conditions of the Creative Commons Attribution (CC BY) license (https:/ / creativecommons.org/licenses/by/ $4.0 /)$.
6 Department of Food Engineering, Faculty of Food Science and Technology, University of Agricultural Sciences and Veterinary Medicine Cluj-Napoca, 3-5 Manastur Street, 400372 Cluj-Napoca, Romania

* Correspondence: corneliu.tanase@umfst.ro (C.T.); teodora.coldea@usamvcluj.ro (T.E.C.); Tel.: +40-074-4215543 (C.T.); +40-746-170337 (T.E.C.)

+ These authors share the first authorship.

Abstract: The influence of the addition of oak chips and barrel ageing on basic wine parameters and volatile compounds of Chardonnay wines has been studied. Chardonnay wines were obtained by the traditional wine-making process. Oak chips ( $4 \mathrm{~g} / \mathrm{L}$-non-toasted and light toasted) were added at the final stage of the winemaking process for ageing 1,2 and 3 months, respectively. Also, the control wine was aged in non-toasted barrels for the same period of time. Following Liquid-liquid extraction-gas chromatography-mass spectrometry analysis, alcohols, esters, fatty acids, lactones, and phenolic compounds were identified and quantified. The light toasted wine was clearly separated by phenolic compounds (vanillin, $p$-vinyl guaiacol and acetovanillone). The floral aroma supplied by 2-phenylethanol was slowly increased by ageing with odor activity values (OAV) higher in aged samples than control wine (1.07). The vanilla scent could be easily perceived in all aged samples, mainly for light toasted chip-treated samples with OAV values between 2.30 and 2.37. After 3 months, the volatile compounds of wine from non-toasted medium (chips and barrels) were almost similar from the volatile profile point of view. This could have economic and vinification management implications since oak barrels are expensive and the wine oak barrel aging is a long process. All wines studied in this research can provide a viable alternative to young varietal wines.

Keywords: Chardonnay wine; chips; barrels; Quercus robur; volatile compounds; light toast

\section{Introduction}

The quality of a wine depends mainly on its chemical composition and also on the expectation of the consumer. Each type of consumer will define wine quality differently depending on their expectations and needs [1]. Expectations of the consumer vary depending on the type of wine: white, red, rose, sweet, dry; young or aged wine [2]. Chardonnay 
is one of the flexible varieties which could adapt to different growth regions with varied weather and soil characteristics [3]. Among white wines, Chardonnay lends itself to a wide variety of production styles, which can be tailored to the target market and that make this grape variety unique. Presently, two tendencies exist for Chardonnay aroma profiles: fruity and light styles and flavored and complex styles [3]. These styles are targeted by current market trends and popularity of wines. Chardonnay allows winemakers different stylistic choices compared to other white wine varieties. Wines can be aged in oak (barrel or chips) and stored after bottling for different periods of time, depending on the desired style [4]. Oak (Quercus sp.) wood is used in the manufacture of barrels due to its chemical and physical properties. Among the other tree species used in winemaking, the oak is unique due to the size and shape of the annual circles that give it durability when transformed into barrel staves [5]. The technological processes used in carpentry, such as seasoning and toasting the wood staves, give the final characteristics of the barrels [6]. Maturing wines in oak barrels is a traditional practice used to improve sensory and quality characteristics. Although fermentation and maturation in oak barrels is desirable for many wine centers, this is not always possible due to high costs $[7,8]$. However, an alternative of obtaining wines with significant aromas is the use of oak, non-toasted or toasted (different levels) chips that can be added during the fermentation process of the wine or at the end of alcoholic fermentation $[7,9,10]$. Some studies examine the addition of wood chips in wine during or after alcoholic fermentation $[7,10]$. Also, the presence of lees during ageing can improve the organoleptic characteristics of some wines [11]. Recently, the use of alternative vessels to oak barrels during winemaking has become increasingly popular, but little is known about their impact on the chemical composition of the resulting wines [12].

The volatile compounds extracted from the wood depend on a number of factors such as the degree of toasting, the contact duration with wine $[13,14]$, but also on their geographical origin [5,15]. The addition of oak chips to finished wines and their effect on the chemical composition of the wine and its sensory characteristics has been widely studied $[3,7,9]$. The effects of the chips addition in the alcoholic fermentation of wine led to contradictory results regarding the stabilization of the wine color, but all studies reported changes in the content of volatile and phenolic compounds $[5,10,13,15]$. Red wine maturation in barrels has been a common practice for centuries. In recent years, the maturation of white wines encountered an increased interest in obtaining wines with improved sensory and quality properties. In Europe, the use of oak wood pieces in winemaking was approved in 2006 (Council Regulation EC No.2165/2005) [16] and since then the addition of oak chips has been generalized. The new EU Delegated Regulation 2019/934 currently regulates the use of oak wood chips in wine practices, which are used for several technological purposes. The main objective is the release of desirable compounds, such as aromas and polyphenolics, from the wood fragments into the wine during aging [16]. The volatile compositions of Chardonnay wines aged in barrels or with chips is studied extensively in Spain [17,18], Greece [19], Italy [20], Australia [21]. Our research is the first attempt to study the volatile composition of Romanian Chardonnay wines, aged through different methods. Chardonnay is one of the grapevine varieties most distributed across the world [17], but in Romania, Chardonnay variety is planted on 2511 hectares representing about $3 \%$ of total vineyards [22].

The purpose of the present study was to examine the effects of two types of French oak chips (non-toasted and light toasted) and non-toasted Romanian oak barrel, during short ageing periods on Chardonnay white wine composition by using gas chromatography coupled to mass spectrometry (GC-MS). Unlike most other white wine varieties which are not aged, Chardonnay wines commonly spend between several weeks to a year for ageing in oak barrels. We focused on identifying the chemical compounds according to contact time and wood type. This study provides important scientific content to winemakers. 


\section{Materials and Methods}

\subsection{Oak Wood Origin}

The barrels used were made up of Romanian oak from Quercus robur, from the forest located in the Western Romania (Ineu area; $46^{\circ} 26^{\prime} \mathrm{N} 21^{\circ} 50^{\prime} \mathrm{E}$ ). The raw staves $(100 \mathrm{~cm} \times 11 \mathrm{~cm} \times 2.2 \mathrm{~cm})$ were naturally seasoned for 24 months at the Transylvania Bois $^{\circledR}$ Tonnellerie (Sighetu Marmației, Romania). Once assembled, the barrels (225 L) were not submitted to toasting procedures. For the purpose of the study, non-toasted barrels were provided to the wine cellar. The oak chips (Quercus robur) were provided by Sodinal ${ }^{\circledR}$ (Wine and Beverage Division of AVeX Group, Paris, France), with dimensions of $0.6 \mathrm{~cm} \times 1.2 \mathrm{~cm} \times 0.15 \mathrm{~cm}$ (width $\times$ length $\times$ thickness). The oak chips used were non-toasted and light toasted, respectively.

\subsection{White Wine Vinification and Sample Collection}

Chardonnay grapes (Vitis vinifera L.) were manually harvested at maturity in Teaca winery (Lechinta, Bistrita Nasaud County, Romania) during the 2017 vintage (225 g/L sugar in grape juice, $8.95 \mathrm{~g} / \mathrm{L}$ must total acidity expressed as tartaric acid and $104 \mathrm{~g}$ weight of 100 berries). The Lechinta grapevine growth area is known for the high acidity of wines, due to climatic conditions (oenoclimatic aptitude index of 4221) [23]. Grapes were crushed and destemmed (Enoitalia ${ }^{\circledR}$ WE223S, Cerreto, Italy) on the harvest day. A pneumatic press (Vaslin-Bucher ${ }^{\circledR}$ RPS 50, France) was used, filled at $75-80 \%$ of its capacity. Potassium metabisulphite $(4.5 \pm 0.5 \mathrm{~g} / \mathrm{hL})$ was added during the transfer of the must to the stainless steel tank (5000 L capacity). Saccharomyces cerevisiae (Zymaflore VL1, Laffort ${ }^{\circledR}$, Bordeaux, France), a commercial active dry yeast was included at a rate of $20 \mathrm{~g} / \mathrm{hL}$ to perform alcoholic fermentation at $16{ }^{\circ} \mathrm{C}$. Malolactic fermentation (MLF) could take place spontaneously, no bacterial culture was added to performed MLF, and no supplementary sulfitation was made to inhibit the MLF. Three sets of experiments were performed depending on the container in which maturation took place (Table 1). When alcoholic fermentation ended, the control wine was transferred to new non-toasted oak barrels at full capacity (first experiment). The control wine was transferred to demijohns (glass container of $50 \mathrm{~L}$ ) with $4 \mathrm{~g} / \mathrm{L}$ non-toasted oak chips (second experiment) and $4 \mathrm{~g} / \mathrm{L}$ light-toasted oak chips (third experiment). Wines were kept in oak barrels and glass containers with chips for ageing during 3 months at a controlled temperature of $14-16^{\circ} \mathrm{C}$. Sampling was performed at 1, 2 and 3 months of wood contact. Wine samples were stored at $4{ }^{\circ} \mathrm{C}$ until the moment of analysis. Each experiment had three sets of containers, meaning three non-toasted barrels, three glass containers with light toasted chips, and three glass containers with non-toasted chips.

Table 1. Experimental design.

\begin{tabular}{cccc}
\hline $\begin{array}{c}\text { Time of Maturation } \\
\text { (Months) }\end{array}$ & Type of Vessel Maturation & Oak Chips (4 g/L) & Abbreviations \\
\hline \multirow{2}{*}{1} & Demijohns/Glass & Non-toasted & N1M \\
& Barrel & Non-toasted & L1M \\
& BAR1M \\
\hline \multirow{2}{*}{2} & Demijohns/Glass & Non-toasted & N2M \\
& Barrel & Non-toasted & L2M \\
& Demijohns/Glass & Non-toasted & N3M \\
\hline \multirow{2}{*}{3} & Barrel & Light-toasted & L3M \\
& Control Wine-unaged wine & & BAR3M \\
\hline
\end{tabular}

\subsection{Oenological Parameters in Wines}

Basic oenological parameters of wines such as $\mathrm{pH}$, alcoholic strength (\% $v / v)$, titratable and volatile acidity ( $\mathrm{g} / \mathrm{L}$ tartaric acid, and $\mathrm{g} / \mathrm{L}$ acetic acid, respectively), were analyzed 
in accordance with official International Organisation of Vine and Wine methods [24] and Bora et al. [25]. All parameters were determined in triplicate.

\subsection{Reagents and Standards}

The reference standards, 2-phenyl ethanol, ethyl decanoate, butyrolactone, isoamyl alcohol and 1-octanol (used as internal standard) were provided by Fluka ${ }^{\circledR}$ (Buch, Switzerland). Dichloromethane used for the extraction of volatile compounds was purchased from Merck (Darmstadt, Germany) Standard stock solutions were prepared by dissolving $10 \mathrm{mg}$ of each reference compound in $10 \mathrm{~mL}$ of dichloromethane. The calibration curve of 2-phneyl-ethanol was used for the volatile concentration calculation. The range of standard concentration was $64-12,900 \mu \mathrm{g} / \mathrm{L}$.

\subsection{Liquid-Liquid Extraction of Volatile Compounds}

The extraction method elaborated by Andujar-Ortiz et al. [26] was adapted and used for the isolation of volatile compounds from wine samples. Into an Erlenmayer flask were introduced $50 \mathrm{~mL}$ of wine spiked with 1-octanol (concentration $614 \mu \mathrm{g} / \mathrm{L}$ ) as the internal standard and $20 \mathrm{~mL}$ of dichloromethane, and it was equipped with a cork. The extraction was performed under continuous stirring in an ice bath for $30 \mathrm{~min}$. The mixture was then kept for $15 \mathrm{~min}$ in an Elmasonic $\mathrm{S}$ ultrasonic bath (Elma, Germany) at the same temperature to avoid the formation of an emulsion. After separation, the organic layer was dried over $\mathrm{Na}_{2} \mathrm{SO}_{4}$, evaporated under a stream of nitrogen to about $200 \mu \mathrm{L}$ volume of the extract. From this solution, $1 \mu \mathrm{L}$ was injected into the GC/MS system. All extractions were performed in triplicate.

\subsection{Determination of Volatile Compounds}

Analysis of volatile organic compounds [27] from wine samples was performed using a Shimadzu QP 2010 PLUS Mass Spectrometer coupled with Gas Chromatograph (Shimadzu, Japan) equipped with a Carbowax type column from Agilent $(30 \mathrm{~m} \times 0.32 \mathrm{~mm}$ inner diameter and $0.50 \mu \mathrm{m}$ film thicknesses). Helium (6.0) was used as carrier gas with a flow rate $1.7 \mathrm{~mL} / \mathrm{min}$. The injector, the detector and the interface temperature were set at $220^{\circ} \mathrm{C}$. The column temperature program was conducted as follows: $40^{\circ} \mathrm{C}$ was the initial temperature for $5 \mathrm{~min}$, increasing at a rate of $4{ }^{\circ} \mathrm{C} / \mathrm{min}$ to $220^{\circ} \mathrm{C}$, and holding $220^{\circ} \mathrm{C}$ for $15 \mathrm{~min}$. Quadrupole mass detector acquisition was carried out using the positive electronic ionization-mode at the $70 \mathrm{eV}$, with continuous scanning from 40 to $500 \mathrm{amu}$. Standard compounds in wines were identified by comparing their relative retention times and mass fragmentation with those of computer matching against a commercial library (National Institute of Standards and Technology and Willey).

\subsection{Odor Activity Values (OAVs)}

The contribution of each volatile compound to wine aroma was evaluated qualitatively by its associated descriptor and quantitatively by its odor activity values (OAVs). OAVs were calculated using the ratio between total concentration (in $\mu \mathrm{g} / \mathrm{L}$ ) of each compound in the wine samples, and the odor threshold value (in $\mu \mathrm{g} / \mathrm{L}$ ) of the compound in water/ethanol solution [28]; threshold values were obtained from information available in the literature [28].

\subsection{Statistical Analysis}

Univariate analysis was performed using analysis of variance (ANOVA), applying the Tukey multiple range test. Partial least squares regression (PLSR) analysis was also carried out, using the XLSTAT Addinsoft 2014.5.03 version (Addinsoft Inc., NY, USA). 


\section{Results}

\subsection{Basic Oenological Parameters}

Analysis of basic oenological parameters is presented in Table 2. Alcohol content is high in all samples (above $12.5 \% \mathrm{v} / \mathrm{v}$ ), with small but significant differences among variants, with a lower value after ageing in barrel. Samples BAR1M-BAR3M aged in non-toasted oak barrels have a higher level of total acidity than samples N1M-N3M and L1M-L3M, probably due to higher oxygen diffusion through barrel wood pores [29]. All tested wine samples reached dryness (less than $4.0 \mathrm{~g} / \mathrm{L}$ residual sugar-data not shown). Nevertheless, there is a small and not significant difference in the dry-extract and non-reducing dry-extract level between the control wine and aged variants (Table 2). The concentration of extract without sugar is very similar in all samples regardless of the ageing method, as previously showed by other studies $[11,30]$. Concentration of free and total $\mathrm{SO}_{2}$ in samples is acceptable for all variants.

\subsection{Volatile Profile of White Wines Aged with Non-Toasted and Light-Toasted Oak Chips}

Liquid-liquid extraction-gas chromatography-mass spectrometry analysis of Chardonnay white wines after 1,2 and 3 months of ageing with non-toasted oak chips, light-toasted oak chips and non-toasted oak barrels, identified five chemical groups: higher alcohols, esters, volatile fatty acids, lactones and phenolic compounds. As noted in Table 3, volatile compounds have significant differences depending on the experimental variant.

\subsubsection{Alcohols}

In our study, the concentration of volatile alcohols varied both depending on the time and type of ageing. Three major alcohols (iso-butanol, isopentyl alcohol and 2phenylethanol) were quantified in wine samples. Those alcohols showed different trends during ageing. As can be observed in Table 3, the iso-butanol significantly decreased during 3 months of ageing, regardless of the methods, while isopentyl alcohol and 2phenylethanol have increased concentration. After 3 months of ageing, the major alcohol concentrations were higher, regardless of the method (N3M-142 mg/L; L3M-144 mg/L and BAR3M-143 mg/L). By using LLE-GC-MS analysis were detected and quantified 9 minor alcohols (hexanol, 2,3-butanediol, 3-methylthio-1-propanol, benzyl-alcohol). In the minor group, hexanol was the highest alcohol, and its concentration was dependent on ageing time and significantly dependent on ageing method. As can be observed from Table 3, the non-toasted barrel ageing method favored the concentration of hexanol, which supplied a leafy and grassy note to the wine samples [31]. Also, the minor alcohols had different trends during ageing. Most of them (4 methyl-1-pentanol, 2-nonalol, 1-heptanol, 3 methylthio-1-propanol) decreased after 3 months of ageing. The highest increase was detected for 2,3-butanediol in BAR1M-BAR3M variants (1686-4973 $\mu \mathrm{g} / \mathrm{L})$.

\subsubsection{Esters}

Individual fruity volatile concentrations obtained in the present research were higher than the bibliographic ranges reported by Gambetta et al. [3] in Italy, or González-Centeno et al. [19], in Greece, but in agreement with those obtained by Cheng et al. [31] in Xianjiang (China). Most of them exhibited concentrations above their olfactory perception threshold, providing the wine with pear, apple, pineapple and/or floral notes [30,31]. Among the ethyl esters of straight-chain fatty acids, ethyl caproate (3120-3240 $\mu \mathrm{g} / \mathrm{L})$ and ethyl octanoate $(2122-2362 \mu \mathrm{g} / \mathrm{L})$ were the predominant ones. Isoamyl acetate, characterized by banana flavor, was the main component among the higher alcohol acetates, with values ranging from 2433 to $2538 \mu \mathrm{g} / \mathrm{L}$, depending on time and method of ageing. Within this family of esters, it was the only volatile present at above threshold level $(30 \mu \mathrm{g} / \mathrm{L})$. 


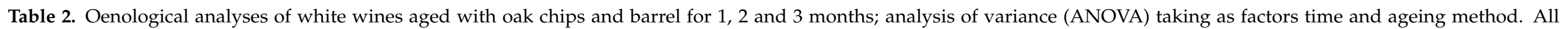

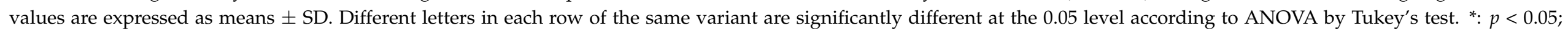

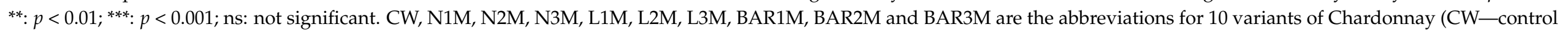
wine, $\mathrm{N}$-non-toasted chips, $\mathrm{L}$-light toasted chips, BAR—non-toasted barrel; $1 \mathrm{M}, 2 \mathrm{M}$ and 3M-number of months of ageing).

\begin{tabular}{|c|c|c|c|c|c|c|c|c|c|c|c|c|}
\hline $\begin{array}{l}\text { Variants/Oenologic } \\
\text { Parameters }\end{array}$ & $\mathrm{CW}$ & N1M & N2M & N3M & L1M & L2M & L3M & BAR1M & BAR2M & BAR3M & Time & $\begin{array}{l}\text { Ageing } \\
\text { Method }\end{array}$ \\
\hline Ethanol $(\% v / v)$ & $12.77 \pm 0.03 a$ & $12.75 \pm 0.04 \mathrm{a}$ & $12.69 \pm 0.08 \mathrm{ab}$ & $12.66 \pm 0.06 b c$ & $12.75 \pm 0.04 a$ & $12.69 \pm 0.08 \mathrm{ab}$ & $12.66 \pm 0.06 \mathrm{bc}$ & $12.62 \pm 0.02 \mathrm{bc}$ & $12.54 \pm 0.04 \mathrm{c}$ & $12.55 \pm 0.02 c$ & $* *$ & $* * *$ \\
\hline $\begin{array}{l}\text { Volatile acidity } \\
\text { (g/L acetic acid) }\end{array}$ & $0.22 \pm 0.02 \mathrm{c}$ & $0.24 \pm 0.03 c$ & $0.28 \pm 0.02 b c$ & $0.31 \pm 0.01 \mathrm{~b}$ & $0.24 \pm 0.03 c$ & $0.28 \pm 0.02 b c$ & $0.31 \pm 0.01 \mathrm{~b}$ & $0.32 \pm 0.02 b$ & $0.41 \pm 0.04 \mathrm{a}$ & $0.46 \pm 0.02 \mathrm{a}$ & $* * *$ & $* * *$ \\
\hline $\begin{array}{l}\text { Total acidity }(\mathrm{g} / \mathrm{L} \\
\text { tartaric acid) }\end{array}$ & $7.96 \pm 0.07 a$ & $7.86 \pm 0.04 \mathrm{ab}$ & $7.74 \pm 0.04 \mathrm{bc}$ & $7.58 \pm 0.08 c$ & $7.84 \pm 0.03 \mathrm{ab}$ & $7.73 \pm 0.04 b c$ & $7.58 \pm 0.08 \mathrm{c}$ & $7.91 \pm 0.04 a$ & $7.87 \pm 0.04 \mathrm{ab}$ & $7.76 \pm 0.07 \mathrm{bc}$ & $* * *$ & $* *$ \\
\hline Dry extract $(\mathrm{g} / \mathrm{L})$ & $23.60 \pm 0.23 a$ & $23.52 \pm 0.35 \mathrm{a}$ & $23.41 \pm 0.39 \mathrm{a}$ & $23.37 \pm 0.25 a$ & $23.52 \pm 0.35 a$ & $23.41 \pm 0.42 \mathrm{a}$ & $23.36 \pm 0.33 a$ & $23.52 \pm 0.31 \mathrm{a}$ & $23.41 \pm 0.40 \mathrm{a}$ & $23.37 \pm 0.35 a$ & ns & ns \\
\hline $\begin{array}{l}\text { Non-reducing dry } \\
\text { extract }(\mathrm{g} / \mathrm{L})\end{array}$ & $22.03 \pm 0.03 a$ & $22.01 \pm 0.04 a$ & $22.05 \pm 0.04 \mathrm{a}$ & $21.99 \pm 0.04 a$ & $22.06 \pm 0.05 a$ & $20.00 \pm 0.03 a$ & $21.92 \pm 0.04 \mathrm{a}$ & $22.04 \pm 0.09 \mathrm{a}$ & $22.07 \pm 0.12 \mathrm{a}$ & $20.05 \pm 0.06 a$ & ns & ns \\
\hline Free $\mathrm{SO}_{2}(\mathrm{mg} / \mathrm{L})$ & $36 \pm 1.00 \mathrm{a}$ & $33 \pm 0.58 \mathrm{ab}$ & $32 \pm 0.58 b c$ & $31 \pm 1.00 \mathrm{c}$ & $34 \pm 0.81 \mathrm{ab}$ & $33 \pm 0.72 b c$ & $30 \pm 0.50 c$ & $32 \pm 1.42 b c$ & $30 \pm 1.15 c$ & $27 \pm 1.05 \mathrm{~d}$ & $* *$ & $* * *$ \\
\hline Total $\mathrm{SO}_{2}(\mathrm{mg} / \mathrm{L})$ & $120 \pm 0.76 b c$ & $123 \pm 2.00 \mathrm{ab}$ & $124 \pm 1.53 a$ & $126 \pm 1.85 a$ & $123 \pm 1.44 a b$ & $124 \pm 2.08 \mathrm{a}$ & $126 \pm 1.76 \mathrm{a}$ & $118 \pm 0.76 \mathrm{~cd}$ & $116 \pm 0.85 \mathrm{~cd}$ & $115 \pm 0.83 \mathrm{~d}$ & * & $* * *$ \\
\hline $\mathrm{pH}$ & $3.22 \pm 0.02 b$ & $3.23 \pm 0.02 \mathrm{ab}$ & $3.23 \pm 0.01 \mathrm{ab}$ & $3.24 \pm 0.01 \mathrm{a}$ & $3.22 \pm 0.02 b$ & $3.23 \pm 0.01 \mathrm{ab}$ & $3.24 \pm 0.02 a$ & $3.23 \pm 0.02 \mathrm{ab}$ & $3.25 \pm 0.03 a$ & $3.24 \pm 0.01 \mathrm{a}$ & * & ns \\
\hline
\end{tabular}




\subsubsection{Fatty Acids}

Nine fatty acids were identified in all wine samples. In the present study, there were significant differences in the fatty acid content between control wine and the wine samples aged with different methods. Furthermore, the concentrations of all kinds of fatty acid detected in the variant N1M-N3M $(4728-5071 \mu \mathrm{g} / \mathrm{L})$ and L1M-L3M $(4336-4977 \mu \mathrm{g} / \mathrm{L})$ were lower compared to variant BAR1M-BAR3M (5035-6540 $\mu \mathrm{g} / \mathrm{L})$.

\subsubsection{Lactones}

Among lactones, butyrolactone showed the highest concentration and depending significantly on the studied factors, as in the case of pantolactone and 3,4-dimethyl-2(5)furanone. Thus, for control sample, an amount of $478 \mu \mathrm{g} / \mathrm{L}$ of total lactones was quantified. Regardless of the ageing method, wines in which non-toasted oak chips were added (N1MN3M) and in non-toasted oak barrel (BAR1M-BAR3M) presented greater butyrolactone concentrations than the corresponding light-toasted chips (L1M-L3M) wines.

\subsubsection{Volatile Phenols}

The total content of phenolic volatiles in Chardonnay wines, calculated by adding up the individual concentration of each compound, ranged from 365 to $463 \mu \mathrm{g} / \mathrm{L}$ for N1M-N3M wines, from 393 to $505 \mu \mathrm{g} / \mathrm{L}$ for L1M-L3M wines and from 350 to $468 \mu \mathrm{g} / \mathrm{L}$ for BAR1M-BAR3M. Among methods of ageing, light toasting led to the highest values, and non-toasted to the lowest ones. Volatile phenols that resulted from wood toasting, have significantly higher values in the wines of L1M-L3M variants ( $p$-vinyl guaiacol58-65 $\mu \mathrm{g} / \mathrm{L}$, acetovanillone- $143-171 \mu \mathrm{g} / \mathrm{L}$ and vanilla $133-139 \mu \mathrm{g} / \mathrm{L})$, compared to the variants from non-toasted wood.

\subsection{Odor Active Odorants}

In order to assess the influence of each single volatile compound, the odor activity value $(\mathrm{OAV})$ was calculated. The $\mathrm{OAV}$ represents the ratio between the concentration of the compound and its odor threshold. Results in Table 4 show that 17 out of 42 quantified volatile compounds reached a concentration above the odor threshold in at least one variant $(\mathrm{OAV}>1)$. For major alcohols, all wine samples presented an $\mathrm{OAV}>1$, with the highest values for isopentyl alcohol, mainly for L1M-L3M sample. The floral aroma supplied by 2-phenylethanol was slowly increased by ageing with OAV higher in aged samples than control wine (1.07). Among minor alcohols, only 2-nonanol and 3-methylthio-1-propanol reached OAV $>1$ in at least one sample. An important ester present above its threshold in all samples was ethyl hexanoate, which can supply anise and strawberry aromas to a wine [19]. $\mathrm{N}$-amyl acetate was found above its threshold in all samples, which indicates that this compound is a general contributor to the fruity aroma of wines. Interestingly for $\mathrm{N}$-amyl acetate was that it increased the OAV values for L1M-L3M (83.80-86.76) samples, so a fruity character could be highlighted by ageing with light-toasted chips. Among terpenes, only linalool showed OAV $>1$. Some authors [32] concluded that the high level for terpenes in wines, could explain the floral descriptors. Three of nine fatty acids showed $\mathrm{OAV}>1$ in all variants. The fatty and rancid aroma supplied by isovaleric acid could be easily perceived in non-toasted oak barrel aged sample, due to the low odor threshold of this compound. Butyrolactone and 3,4-dimethyl-2(5)-furanone were lactones with OAV $>1$. Butyrolactone extracted from non-toasted wood presented higher values of OAV for N1M-N3M (9.77-12.66) and BAR1M-BAR3M (13.17-15.20) samples. For volatile phenols, 4 -vinyl guaiacol and vanilla reached $\mathrm{OAV}>1$ in at least one variant. The vanilla scent could be easily perceived in all aged samples, especially in L1M-L3M samples with OAV values between 2.30 and 2.37 . 


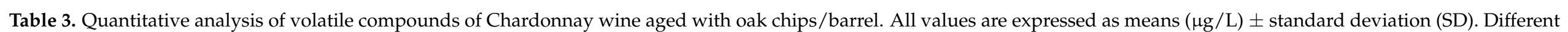

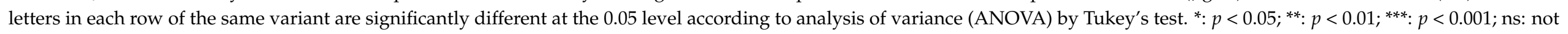

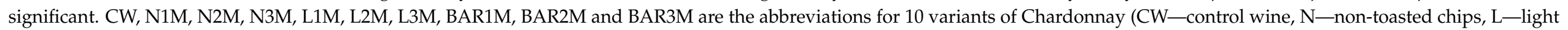
toasted chips, BAR—non-toasted barrel; 1M, 2M and 3M-number of months of ageing).

\begin{tabular}{|c|c|c|c|c|c|c|c|c|c|c|c|c|}
\hline $\begin{array}{l}\text { Variant/Volatile } \\
\text { Compounds }\end{array}$ & $\mathrm{CW}$ & N1M & N2M & N3M & L1M & L2M & L3M & BAR1M & BAR2M & BAR3M & Time & $\begin{array}{l}\text { Ageing } \\
\text { Method }\end{array}$ \\
\hline Isobutanol & $47 \pm 0.03 a$ & $39 \pm 2.7 \mathrm{~d}$ & $40 \pm 3.8 \mathrm{c}$ & $42 \pm 3.5 b$ & $39 \pm 1.7 \mathrm{~d}$ & $41 \pm 2.3 b c$ & $41 \pm 1.9 \mathrm{bc}$ & $38 \pm 2.8 \mathrm{e}$ & $38 \pm 2.6 \mathrm{e}$ & $41 \pm 2.4 \mathrm{bc}$ & $* *$ & $* * *$ \\
\hline Isopentyl alcohol & $76 \pm 2.1 \mathrm{e}$ & $83 \pm 4.5 c$ & $84 \pm 5.2 \mathrm{bc}$ & $83 \pm 5.7 c$ & $85 \pm 4.2 \mathrm{ab}$ & $84 \pm 3.2 b c$ & $86 \pm 5.8 \mathrm{a}$ & $82 \pm 2.1 \mathrm{~d}$ & $83 \pm 3.0 c$ & $84 \pm 2.7 \mathrm{bc}$ & * & $* * *$ \\
\hline 2-phenylethanol & $15 \pm 0.3 \mathrm{e}$ & $16 \pm 2.4 \mathrm{~d}$ & $17 \pm 2.0 \mathrm{c}$ & $17 \pm 2.8 \mathrm{c}$ & $16 \pm 1.9 \mathrm{~d}$ & $18 \pm 2.5 \mathrm{~b}$ & $17 \pm 2.9 \mathrm{c}$ & $19 \pm 2.6 \mathrm{a}$ & $17 \pm 2.1 \mathrm{c}$ & $18 \pm 2.8 \mathrm{~b}$ & * & $* * *$ \\
\hline $\begin{array}{c}\text { Total major alcohols } \\
(\mathrm{mg} / \mathrm{L})\end{array}$ & 138 & 138 & 141 & 142 & 140 & 145 & 144 & 139 & 138 & 143 & & \\
\hline Hexanol & $564 \pm 5.7 \mathrm{de}$ & $438 \pm 3.2 \mathrm{e}$ & $398 \pm 7.8 \mathrm{f}$ & $865 \pm 6.5 b$ & $375 \pm 24 \mathrm{f}$ & $342 \pm 7.8 \mathrm{f}$ & $1057 \pm 50 \mathrm{a}$ & $760 \pm 42 \mathrm{bc}$ & $627 \pm 8.7 \mathrm{c}$ & $1145 \pm 12.1 \mathrm{a}$ & * & $* * * *$ \\
\hline 4-methyl-1-pentanol & $22 \pm 0.6 \mathrm{~b}$ & $25 \pm 2.6 \mathrm{ab}$ & $11 \pm 3 \mathrm{~cd}$ & $12 \pm 0.9 \mathrm{~cd}$ & $31 \pm 1.5 \mathrm{a}$ & $10 \pm 1.8 \mathrm{~d}$ & $9.7 \pm 0.5 \mathrm{~d}$ & $33 \pm 5.7 a$ & $22 \pm 7.8 \mathrm{bc}$ & $18 \pm 2.8 \mathrm{bc}$ & ** & ** \\
\hline E-3-hexenol & $30 \pm 1.2 \mathrm{~b}$ & $30 \pm 3.5 b$ & $16 \pm 3.1 \mathrm{c}$ & $17 \pm 1.5 \mathrm{c}$ & $40 \pm 6.6 \mathrm{a}$ & $41 \pm 3.5 \mathrm{a}$ & $41 \pm 3.3 a$ & $27 \pm 1.2 \mathrm{~b}$ & $24 \pm 3.5 \mathrm{bc}$ & $45 \pm 3.4 a$ & * & $* *$ \\
\hline 2-nonanol & $173 \pm 9.6 a$ & $100 \pm 11 b$ & $47 \pm 4.5 \mathrm{de}$ & $58 \pm 6.8 \mathrm{de}$ & $115 \pm 3.5 b$ & $37 \pm 5.2 \mathrm{e}$ & $59 \pm 6.8 \mathrm{de}$ & $98 \pm 2.1 \mathrm{c}$ & $78 \pm 7.8 \mathrm{~cd}$ & $13 \pm 0.6 \mathrm{f}$ & $* *$ & $* * *$ \\
\hline 1-heptanol & $124 \pm 1.2 \mathrm{e}$ & $230 \pm 9.4 a$ & $201 \pm 4.9 \mathrm{bc}$ & $174 \pm 3.1 \mathrm{~d}$ & $240 \pm 35 a$ & $150 \pm 22 \mathrm{~d}$ & $136 \pm 6.0 \mathrm{c}$ & $230 \pm 5.0 \mathrm{a}$ & $211 \pm 2.6 \mathrm{~b}$ & $182 \pm 3.7 \mathrm{c}$ & $* * *$ & $* * *$ \\
\hline 2,3-butanediol & $301 \pm 35 \mathrm{e}$ & $507 \pm 24 \mathrm{de}$ & $1137 \pm 12.9 \mathrm{~cd}$ & $1417 \pm 21 \mathrm{c}$ & $330 \pm 27 \mathrm{e}$ & $605 \pm 9.8 \mathrm{de}$ & $769 \pm 45 \mathrm{de}$ & $1686 \pm 14.7 \mathrm{c}$ & $2797 \pm 35.2 \mathrm{~b}$ & $4973 \pm 52 \mathrm{a}$ & $* * *$ & $* * *$ \\
\hline $\begin{array}{l}\text { 3-methylthio-1- } \\
\text { propanol }\end{array}$ & $278 \pm 15 b c$ & $248 \pm 15 b c$ & $250 \pm 25 b c$ & $206 \pm 19 d$ & $176 \pm 15 \mathrm{~d}$ & $166 \pm 31 d$ & $222 \pm 17 b c$ & $553 \pm 49 a$ & $290 \pm 17 b$ & $244 \pm 75 b c$ & $* * *$ & $* * *$ \\
\hline Benzylalcohol & $58 \pm 3.5 \mathrm{a}$ & $44 \pm 2.4 c$ & $45 \pm 1.90 c$ & $47 \pm 2.2 \mathrm{bc}$ & $42 \pm 2.6 \mathrm{~d}$ & $49 \pm 2.4 b$ & $48 \pm 3.1 b$ & $32 \pm 2.4 \mathrm{f}$ & $34 \pm 2.1 \mathrm{e}$ & $39 \pm 3.5 \mathrm{de}$ & $* * *$ & $* * *$ \\
\hline $\begin{array}{c}\text { Total minor alcohols } \\
(\mu \mathrm{g} / \mathrm{L})\end{array}$ & 1662 & 1741 & 2222 & 2917 & 1460 & 1514 & 2454 & 3541 & 4207 & 6782 & & \\
\hline Linalool & $323 \pm 7.8 \mathrm{a}$ & $59 \pm 4.9 c$ & $49 \pm 4.6 \mathrm{~cd}$ & $33 \pm 2.8 \mathrm{e}$ & Trace & trace & Trace & $83 \pm 7.3 b$ & $45 \pm 2.3 \mathrm{de}$ & $36 \pm 2.3 e$ & $* * *$ & $* * *$ \\
\hline Total terpenes $(\mu \mathrm{g} / \mathrm{L})$ & 502 & 81 & 49 & 33 & trace & trace & Trace & 120 & 55 & 47 & & \\
\hline Isobutyric acid & $83 \pm 3.7 b$ & $81 \pm 2.5 b c$ & $51 \pm 4.6 \mathrm{de}$ & $21 \pm 2.1 f$ & $108 \pm 10 a$ & $83 \pm 3.1 b$ & $39 \pm 4.0 \mathrm{e}$ & $75 \pm 3.3 \mathrm{bc}$ & $64 \pm 5.6 \mathrm{~cd}$ & $24 \pm 2.8 \mathrm{f}$ & $* * *$ & $* * *$ \\
\hline Hexanoic acid & $945 \pm 17 \mathrm{e}$ & $1267 \pm 21 \mathrm{~d}$ & $1643 \pm 68 \mathrm{a}$ & $1395 \pm 45 c$ & $1640 \pm 96 a$ & $1227 \pm 21 \mathrm{e}$ & $1355 \pm 40 \mathrm{c}$ & $1574 \pm 96 \mathrm{~b}$ & $1501 \pm 65 b c$ & $1016 \pm 10 \mathrm{~d}$ & $* * *$ & $* * *$ \\
\hline Isovaleric acid & $132 \pm 2.1 \mathrm{e}$ & $156 \pm 9.6 \mathrm{~d}$ & $219 \pm 82 b c$ & ND & $163 \pm 9.5 \mathrm{~d}$ & $189 \pm 15 b c$ & $190 \pm 15 b c$ & $229 \pm 7.4 \mathrm{bc}$ & $296 \pm 19 b$ & $538 \pm 4.4 a$ & $* *$ & $* * *$ \\
\hline Lactic acid & $54 \pm 4.7 \mathrm{e}$ & $132 \pm 21 \mathrm{~d}$ & $228 \pm 19 \mathrm{e}$ & $417 \pm 67 c$ & $141 \pm 5.8 \mathrm{~d}$ & $192 \pm 32 d$ & $108 \pm 10 \mathrm{e}$ & $431 \pm 17 c$ & $808 \pm 75 b$ & $1317 \pm 145 a$ & $* * *$ & $* * *$ \\
\hline Octanoic acid & $2459 \pm 58 c$ & $2377 \pm 165 b c$ & $2448 \pm 39 c$ & $2653 \pm 72 b$ & $2054 \pm 89 \mathrm{~d}$ & $2409 \pm 39 c$ & $2442 \pm 54 c$ & $2069 \pm 153 d$ & $2447 \pm 154 c$ & $2866 \pm 75 a$ & ** & $* * *$ \\
\hline Decanoic acid & $957 \pm 26 a$ & $449 \pm 56 b$ & $227 \pm 25 d$ & $125 \pm 9.8 \mathrm{e}$ & $306 \pm 19 \mathrm{~cd}$ & $117 \pm 10 \mathrm{e}$ & $124 \pm 13 \mathrm{e}$ & $441 \pm 41 \mathrm{~b}$ & $274 \pm 30 \mathrm{~cd}$ & $324 \pm 21 c$ & $* * *$ & $* * *$ \\
\hline Malic acid & $571 \pm 62 \mathrm{a}$ & $193 \pm 68 c$ & $176 \pm 2.5 \mathrm{e}$ & $155 \pm 12 \mathrm{~d}$ & $576 \pm 42 \mathrm{a}$ & $208 \pm 51 c$ & $184 \pm 7.2 \mathrm{e}$ & $372 \pm 356 b$ & $205 \pm 24 c$ & $165 \pm 59 \mathrm{~d}$ & $* *$ & $* * *$ \\
\hline $\begin{array}{l}\text { 5-oxotetrahydrofuran2- } \\
\text { carboxilic } \\
\text { acid }\end{array}$ & $71 \pm 1.9 \mathrm{de}$ & $61 \pm 6.3 \mathrm{e}$ & $83 \pm 3.6 \mathrm{~d}$ & $91 \pm 6.8 \mathrm{~cd}$ & $103 \pm 14 b c$ & $116 \pm 9.8 b$ & $126 \pm 32 a$ & $36 \pm 4.0 f$ & $28 \pm 0.9 f$ & $71 \pm 5.2 \mathrm{de}$ & $* *$ & $* * *$ \\
\hline 2-oxoapidic & $8 \pm 0.7 f$ & $12 \pm 1.5 \mathrm{de}$ & $17 \pm 1.9 \mathrm{e}$ & $14 \pm 1.7 \mathrm{~cd}$ & $22 \pm 1.6 \mathrm{a}$ & $19 \pm 1.4 \mathrm{ab}$ & $17 \pm 1.2 \mathrm{~b}$ & $15 \pm 1.5 c$ & $11 \pm 1.8 \mathrm{e}$ & $12 \pm 1.6 \mathrm{de}$ & ** & ** \\
\hline
\end{tabular}


Table 3. Cont.

\begin{tabular}{|c|c|c|c|c|c|c|c|c|c|c|c|c|}
\hline Total fatty acids $(\mu \mathrm{g} / \mathrm{L})$ & 5280 & 4728 & 5071 & 4792 & 4645 & 4336 & 4977 & 5035 & 5634 & 6540 & & \\
\hline $\mathrm{N}$-amyl acetate & $2483 \pm 12 \mathrm{~cd}$ & $2433 \pm 12 d$ & $2487 \pm 40 \mathrm{~cd}$ & $2471 \pm 8 \mathrm{~cd}$ & $2581 \pm 42 b c$ & $2514 \pm 11 b c$ & $2603 \pm 31 a$ & $2462 \pm 41 \mathrm{~cd}$ & $2508 \pm 36 \mathrm{bc}$ & $2548 \pm 52 b c$ & $* *$ & $* * *$ \\
\hline Hexylacetate & $536 \pm 13 a$ & $474 \pm 31 b c$ & $463 \pm 35 c$ & $423 \pm 21 \mathrm{e}$ & $486 \pm 23 b$ & $479 \pm 13 b c$ & $465 \pm 28 c$ & $452 \pm 19 c d$ & $416 \pm 13 e$ & $421 \pm 22$ de & $*$ & * \\
\hline Ethyl hexanoate & $3151 \pm 76 c$ & $3165 \pm 96 b$ & $3213 \pm 43 \mathrm{~d}$ & $3120 \pm 27 b c$ & $3193 \pm 36 b$ & $3160 \pm 62 b$ & $3141 \pm 58 c$ & $3240 \pm 22 \mathrm{a}$ & $3103 \pm 93 d$ & $3128 \pm 71 b c$ & $* * *$ & $* * *$ \\
\hline Ethyl lactate & $395 \pm 15 f$ & $1031 \pm 59 b c$ & $766 \pm 38 \mathrm{~cd}$ & $849 \pm 75 \mathrm{~cd}$ & $1179 \pm 54 \mathrm{~b}$ & $616 \pm 58 \mathrm{e}$ & $659 \pm 78 \mathrm{e}$ & $1010 \pm 64 \mathrm{bc}$ & $954 \pm 87 \mathrm{bc}$ & $2122 \pm 72 a$ & $* * *$ & $* * *$ \\
\hline Ethyl octanoate & $2271 \pm 12 \mathrm{~b}$ & $2148 \pm 34 \mathrm{bc}$ & $2322 \pm 32 a$ & $2297 \pm 46 \mathrm{~b}$ & $2177 \pm 54 \mathrm{bc}$ & $2362 \pm 59 a$ & $2214 \pm 26 c$ & $2122 \pm 27 b c$ & $2131 \pm 12 c$ & $2184 \pm 68 \mathrm{~b}$ & $* * *$ & ns \\
\hline Phenethyl acetate & $334 \pm 15 d$ & $487 \pm 15 b c$ & $530 \pm 30 b$ & $586 \pm 21 a$ & $476 \pm 19 b c$ & $490 \pm 23 b c$ & $517 \pm 45 b$ & $374 \pm 38 c$ & $357 \pm 41 \mathrm{~cd}$ & $506 \pm 11 b c$ & ns & $* * *$ \\
\hline Diethyl malate & $142 \pm 23 g$ & $222 \pm 29 c$ & $286 \pm 29 a$ & $219 \pm 20 c$ & $169 \pm 32 f$ & $162 \pm 9.8 \mathrm{f}$ & $202 \pm 18 \mathrm{e}$ & $256 \pm 54 b$ & $299 \pm 32 \mathrm{a}$ & $282 \pm 49 a$ & $* *$ & $* * *$ \\
\hline Diethyl succinate & $115 \pm 21 \mathrm{~d}$ & $283 \pm 32 \mathrm{ab}$ & $270 \pm 38 b$ & $244 \pm 25 c$ & $285 \pm 41 \mathrm{ab}$ & $265 \pm 21 b$ & $240 \pm 28 c$ & $269 \pm 29 b$ & $286 \pm 21 \mathrm{ab}$ & $294 \pm 72 a$ & $* *$ & $* * *$ \\
\hline Trimethylene acetate & $431 \pm 25 a$ & $363 \pm 12 \mathrm{e}$ & $341 \pm 50 f$ & $358 \pm 6.2 \mathrm{e}$ & $276 \pm 36 c$ & $287 \pm 24 c d$ & $273 \pm 5 \mathrm{de}$ & $369 \pm 35 \mathrm{e}$ & $360 \pm 42 \mathrm{e}$ & $399 \pm 28 b$ & $* * *$ & $* * *$ \\
\hline Total esters $(\mu \mathrm{g} / \mathrm{L})$ & 10133 & 10902 & 10827 & 10738 & 11103 & 10452 & 10464 & 10839 & 10638 & 12038 & & \\
\hline $\begin{array}{l}\text { Butyrolactone } \\
\text { Pantolactone }\end{array}$ & $\begin{array}{l}344 \pm 41 b \\
12 \pm 0.3 b\end{array}$ & $\begin{array}{l}443 \pm 37 \mathrm{ab} \\
\text { trace }\end{array}$ & $\begin{array}{l}369 \pm 27 a b \\
\text { trace }\end{array}$ & $\begin{array}{l}342 \pm 29 \mathrm{ab} \\
\text { trace }\end{array}$ & $\begin{array}{l}228 \pm 21 c \\
11 \pm 3.2 b\end{array}$ & $\begin{array}{l}260 \pm 14.5 b c \\
\text { trace }\end{array}$ & $\begin{array}{l}208 \pm 14 c \\
\text { Trace }\end{array}$ & $\begin{array}{l}532 \pm 18 a \\
\text { trace }\end{array}$ & $\begin{array}{l}466 \pm 12 \mathrm{ab} \\
\text { trace }\end{array}$ & $\begin{array}{c}461 \pm 10 \mathrm{ab} \\
44 \pm 3.6 \mathrm{a}\end{array}$ & $\begin{array}{l}* * \\
*\end{array}$ & * \\
\hline $\begin{array}{l}\text { 3,4-dimethyl-2(5)- } \\
\text { furanone }\end{array}$ & $122 \pm 12 \mathrm{a}$ & $65 \pm 6.7 \mathrm{bc}$ & $43 \pm 3.5 \mathrm{~cd}$ & $52 \pm 4.2 \mathrm{~cd}$ & $84 \pm 9.6 b$ & $33 \pm 3.2 \mathrm{de}$ & $42 \pm 9.2 \mathrm{~cd}$ & $71 \pm 2.1 \mathrm{bc}$ & $69 \pm 7.0 \mathrm{bc}$ & $51 \pm 5.7 \mathrm{~cd}$ & $* * *$ & $* * *$ \\
\hline Total lactones $(\mu \mathrm{g} / \mathrm{L})$ & 478 & 508 & 412 & 394 & 323 & 293 & 250 & 603 & 535 & 556 & & \\
\hline p-vinyl guiacol & $18 \pm 2 c$ & $26 \pm 3.6 b$ & $28 \pm 3.2 b$ & $31 \pm 2.4 b$ & $58 \pm 12 a$ & $61 \pm 12 \mathrm{a}$ & $65 \pm 13 a$ & $26 \pm 6.4 b$ & $29 \pm 3.1 b$ & $33 \pm 4.2 b$ & $*$ & $* * *$ \\
\hline $\begin{array}{c}\text { Methyl- } \\
\text { hydroxycinamate }\end{array}$ & $77 \pm 6.3 a$ & $32 \pm 4.5 b$ & $16 \pm 1.2 \mathrm{~d}$ & $12 \pm 1.3 \mathrm{e}$ & $21 \pm 4.7 \mathrm{c}$ & $14 \pm 1.1 \mathrm{de}$ & $11 \pm 0.9 \mathrm{e}$ & $26 \pm 2.4 b c$ & $13 \pm 1.4 \mathrm{e}$ & $12 \pm 1.2 \mathrm{e}$ & $* * *$ & $* * *$ \\
\hline Acetovanillone & $61 \pm 0.8 \mathrm{e}$ & $157 \pm 21 b$ & $122 \pm 24 \mathrm{~d}$ & $119 \pm 12 d$ & $171 \pm 35 \mathrm{a}$ & $167 \pm 19 a$ & $143 \pm 15 c$ & $162 \pm 32 \mathrm{ab}$ & $139 \pm 21 \mathrm{~cd}$ & $121 \pm 11 d$ & $* * *$ & $* *$ \\
\hline $\begin{array}{c}2,3- \\
\text { hydroxybenzofurane }\end{array}$ & $156 \pm 5.4 a$ & $114 \pm 1.6 \mathrm{c}$ & $63 \pm 5.9 e$ & $79 \pm 1.8 \mathrm{de}$ & $116 \pm 3.1 \mathrm{c}$ & $44 \pm 1.2 \mathrm{f}$ & $33 \pm 1.4 \mathrm{f}$ & $127 \pm 6.3 b$ & $99 \pm 8.5 \mathrm{~d}$ & $56 \pm 2.1 \mathrm{ef}$ & $* * *$ & * \\
\hline Vanillin & $39 \pm 3.5 \mathrm{e}$ & $134 \pm 2.1 \mathrm{c}$ & $136 \pm 1.9 \mathrm{bc}$ & $133 \pm 1.7 \mathrm{c}$ & $139 \pm 2.1 \mathrm{ab}$ & $138 \pm 1.8 \mathrm{~b}$ & $141 \pm 2.3 \mathrm{a}$ & $127 \pm 1.4 \mathrm{~d}$ & $126 \pm 1.1 \mathrm{~d}$ & $128 \pm 1.9 \mathrm{~d}$ & $*$ & $* *$ \\
\hline $\begin{array}{l}\text { Total volatile phenols } \\
(\mu \mathrm{g} / \mathrm{L})\end{array}$ & 351 & 463 & 365 & 374 & 505 & 424 & 393 & 468 & 406 & 350 & & \\
\hline
\end{tabular}




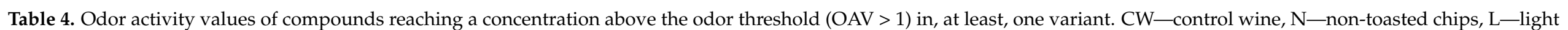
toasted chips, BAR—-non-toasted barrel; 1M, 2M and 3M-number of months of ageing); ODT-olfactory detection threshold.

\begin{tabular}{|c|c|c|c|c|c|c|c|c|c|c|c|c|c|}
\hline Volatile Compounds & Odor Descriptor & $\begin{array}{c}\text { ODT } \\
(\mathrm{mL} \text { or } \mu \mathrm{L})\end{array}$ & References & CW & N1M & $\mathbf{N} 2 \mathrm{M}$ & N3M & L1M & L2M & L3M & BAR1M & BAR2M & BAR3M \\
\hline Iso-butanol (mg/L) & Bitter, fusel, alcohol & 40 & [27] & 1.17 & 0.97 & 1.00 & 1.05 & 0.97 & 1.02 & 1.02 & 0.95 & 0.95 & 1.02 \\
\hline Iso-pentyl alcohol (mg/L) & Fusel & 30 & [32] & 2.53 & 2.76 & 2.80 & 2.76 & 2.83 & 2.80 & 2.86 & 2.73 & 2.76 & 2.80 \\
\hline 2-nonanol $(\mu \mathrm{g} / \mathrm{L})$ & $\begin{array}{l}\text { green creamy, citrus, } \\
\text { cheese, fruity }\end{array}$ & 58 & {$[34]$} & 2.98 & 1.72 & 0.81 & 1.00 & 1.98 & 0.63 & 1.01 & 1.68 & 1.34 & 0.22 \\
\hline $\begin{array}{l}\text { 3-methylthio-1-propanol } \\
(\mu \mathrm{g} / \mathrm{L})\end{array}$ & $\begin{array}{l}\text { Boiled potato, } \\
\text { rubber }\end{array}$ & 500 & [32] & 0.55 & 0.49 & 0.50 & 0.41 & 0.35 & 0.33 & 0.44 & 1.10 & 0.58 & 0.48 \\
\hline Iso-butyric acid $(\mu \mathrm{g} / \mathrm{L})$ & Fatty, butter, cheese & 50 & [32] & 1.66 & 1.62 & 1.02 & 0.42 & 2.16 & 1.66 & 0.78 & 1.5 & 1.28 & 0.48 \\
\hline Isovaleric acid $(\mu \mathrm{g} / \mathrm{L})$ & $\begin{array}{l}\text { Fatty, rancid, } \\
\text { sweaty }\end{array}$ & 33.4 & [33] & 3.95 & 4.67 & 6.55 & - & 4.88 & 5.65 & 5.68 & 6.85 & 8.86 & 16.10 \\
\hline Octanoic acid $(\mu \mathrm{g} / \mathrm{L})$ & $\begin{array}{l}\text { Rancid, cheese, } \\
\text { harsh }\end{array}$ & 500 & [33] & 4.91 & 4.75 & 4.89 & 5.30 & 4.10 & 4.81 & 4.88 & 4.13 & 4.89 & 5.73 \\
\hline $\mathrm{N}$-amyl acetate $(\mu \mathrm{g} / \mathrm{L})$ & Banana & 30 & [32] & 82.76 & 81.10 & 82.9 & 82.36 & 86.03 & 83.80 & 86.76 & 82.06 & 83.60 & 84.93 \\
\hline Ethyl hexanoate $(\mu \mathrm{g} / \mathrm{L})$ & $\begin{array}{l}\text { Apple, anise, } \\
\text { strawberry }\end{array}$ & 5 & [32] & 630.2 & 633.0 & 642.6 & 624.0 & 638.6 & 632.0 & 628.2 & 648.0 & 620.6 & 625.6 \\
\hline Ethyl octanoate $(\mu \mathrm{g} / \mathrm{L})$ & Pineapple, pear & 2 & {$[32]$} & 1135 & 1074 & 1161 & 1148 & 1088 & 1181 & 1107 & 1061 & 1065 & 1092 \\
\hline $\begin{array}{l}\text { 3,4-dimethyl-2(5)-furanone } \\
(\mu \mathrm{g} / \mathrm{L})\end{array}$ & Caramel & 19 & {$[35]$} & 6.42 & 3.42 & 2.26 & 2.73 & 4.42 & 1.74 & 2.21 & 3.74 & 3.63 & 2.68 \\
\hline$p$-vinyl guiacol $(\mu \mathrm{g} / \mathrm{L})$ & Clove, smoke, spice & 40 & [27] & 0.45 & 0.65 & 0.70 & 0.77 & 1.45 & 1.53 & 1.63 & 0.65 & 0.73 & 0.83 \\
\hline Vanillin $(\mu \mathrm{g} / \mathrm{L})$ & Vanilla, sweet & 60 & [35] & 0.65 & 2.23 & 2.27 & 2.22 & 2.37 & 2.30 & 2.35 & 2.12 & 2.10 & 2.13 \\
\hline
\end{tabular}




\subsection{Multivariate Analysis}

Figure 1 shows the chemical analysis results of the Chardonnay wines overlaid over the studied variants, with the wines projected on to that space. According to PLSR analysis, the distance between the variable and the center of the circle shows the interpretive degree of the principal components to the variable. The PLSR was established to determine the influence of methods and duration of ageing on volatile compounds.

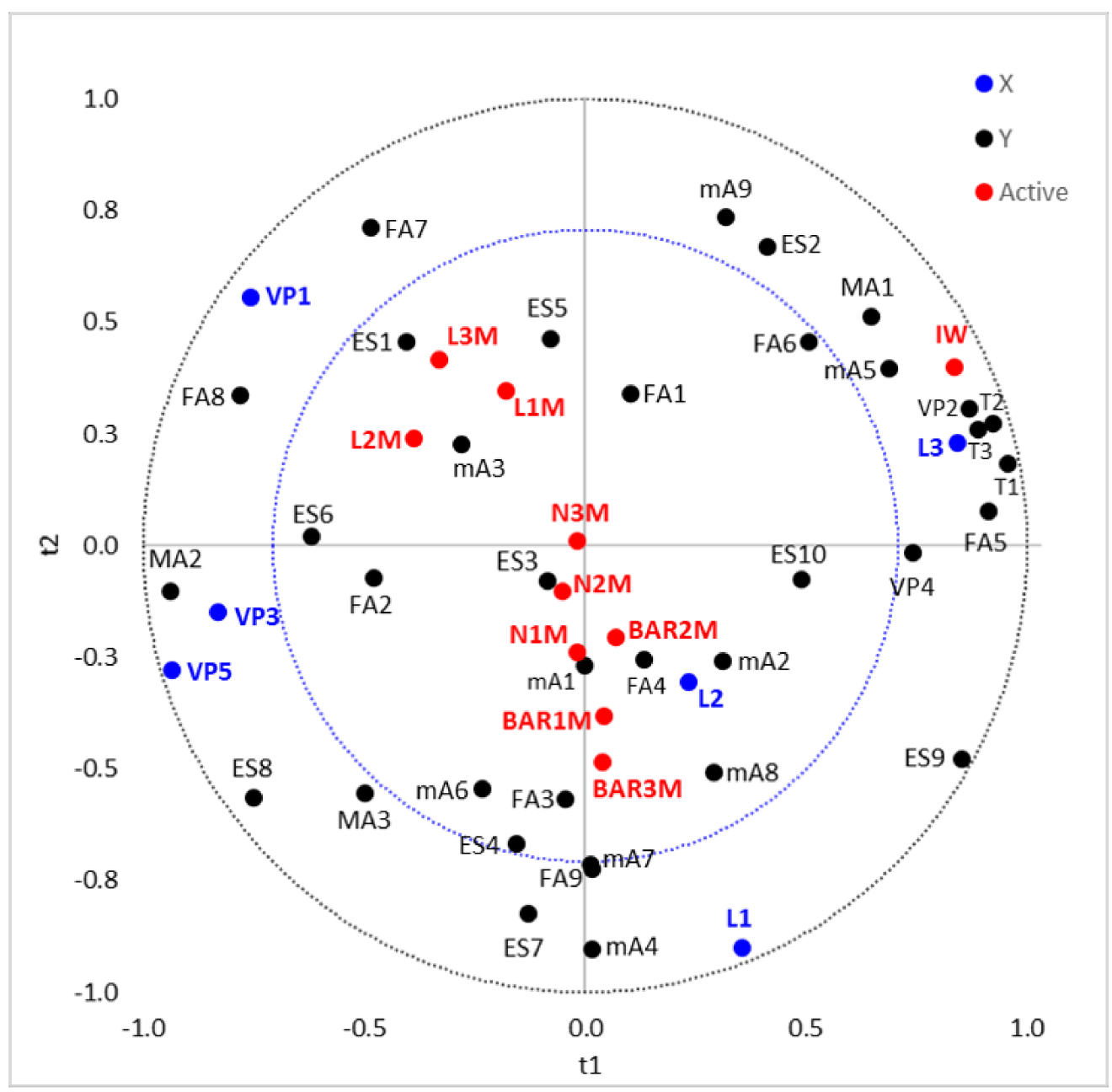

Figure 1. Partial least squares regression (PLSR) analysis, chemical data and the correlation of Chardonnay wines between ageing method and time ( $\mathrm{t} 1$, chemical components of control wine; $\mathrm{t}$, chemical components released from wood). CW, N1M, N2M, N3M, L1M, L2M, L3M, BAR1M, BAR2M and BAR3M are the abbreviations for 10 variants of Chardonnay ( $\mathrm{CW}-$ control wine, $\mathrm{N}$-non-toasted chips, L-light toasted chips, BAR-non-toasted barrel; $1 \mathrm{M}, 2 \mathrm{M}$ and 3M-number of months of ageing). MA1 (iso-butanol), MA2 (isopenthyl), MA3 (2-phenylethanol), mA1 (1-hexanol), mA2 (4-methy-1pentanol), mA3 (E-3-hexenol), mA4 (Z-3-hexenol), mA5 (2-nonanol), mA6 (1-heptanol), mA7 (2,3-butanediol), mA8 (3-methylthio-1-propanol), mA9 (benzyl-alcohol), T1 (linalool), T2 (terpineol), T3 (trans-geraniol), FA1 (iso-butyric acid), FA2 (hexanoic acid), FA3 (isovaleric acid), FA4 (octanoic acid), FA5 (decanoic acid), FA6 (malic acid), FA7 (5-oxotetrahydrofuran-2-carboxilic acid), FA8 (2-oxoapidic), FA9 (lactic acid), ES1 (N-amyl acetate), ES2 (hexyl-acetate), ES3 (ethyl hexanoate), ES4 (ethyl lactate), ES5 (ethyl octanoate), ES6 (phenethyl acetate), ES7 (diethyl malate), ES78 (diethyl succinate), ES9 (tri-methylene acetate), ES10 (ethyl-4-hydroxybutanoate), L1 (butyrolactone), L2 (pantolactone), L3 (3,4-dimethyl-2(5)-furanone), VP1 ( $p$-vinyl guaiacol), VP2 (methyl-hydroxycinamate), VP3 (acetovanillone), VP4 (2,3-hydroxybenzofurane), VP5 (vanillin). 
In Figure 1, the volatile compounds of the control wine can be used to distinguish the aroma compounds of the aged wines. Control wine (unaged) is well related to alcohols such as iso-butanol (MA1), 2-nonanol (mA5), and to the terpenes: linalool (T1), terpineol (T2) and trans-geraniol (T3). Esters like hexyl-acetate (ES2), fatty acids like malic acids (FA6) and lactones like 2,4-dimethyl-2(5)-furanone were also related to the control Chardonnay wine. Those compounds are specific for fresh wines. The wines that pass through the maturation process are related to the esters, fatty acids, lactones and volatile phenols [36,37]. As can be seen from Figure 1, the wines aged in non-toasted medium (chips and barrel) appear as grouped against the wines aged with light toasted chips. This finding is similar with other research by Calugar et al. [13], for Muscat Ottonel wines. The non-toasted barrel ageing is correlated with lactones that came from untreated wood-butyrolactone (L1) and pantolactone (L2). The non-toasted medium is also related to esters such as ethyl lactate (ES4), ethyl caproate (ES3), and carboxylic acid, as lactic acid (FA9), octanoic acid (FA4) and isovaleric acid (FA3). The majority of volatile phenols quantified in this research, came from the thermal treatment of wood and are correlated with wines aged with light-toasted chips. In a study, Farrel et al. [38], stated that vanillin (VP5), p-vinyl guaiacol (VP1) and acetovanillone (VP3) are volatile phenols which come from the thermal degradation of wood.

\section{Discussion}

The diversity of oak products allows winemakers to achieve different results according to the expected outcome and consumer preferences. Exposure to oak wood may cause unbalanced flavor profiles with strong oak aromas if the maturation period is longer [30].

\subsection{Basic Parameters of Chardonnay Aged Wines}

During wood contact, basic parameters of wines were affected. The decrease of alcohol content could be the result of the evaporative loss of ethanol and its diffusion through the staves, which are higher than that of water during ageing [29]. The increase in titratable acidity is due to the rise in volatile acidity; during the first months this rise could indicate the extraction of carboxylic, phenolic and volatile acids from the wood as suggested by Aiken and Noble, [39]. According to Towey and Waterhouse [40], the pH and ethanol of wines also influenced the amount of volatiles extracted from oak products. It has been stated that the characteristics of the wine itself may also be very important during the ageing process, since $\mathrm{pH}$, ethanol and acidity may have a direct influence on ethanolysis of wood components. Some contradictory results have been found. GardeCerdán et al. [41,42] stated that the extraction of furanic aldehydes, oak lactones, phenolic aldehydes and alcohols was influenced by the wine alcohol content, the wine $\mathrm{pH}$ being less significant, whereas Ortega-Heras et al. [43] did not find significant correlations between wine ethanol content and the levels of any of the volatile compounds studied.

\subsection{Volatile Compounds in Chardonnay Aged Wine}

The method of ageing influences the amount of chemical compounds at different times of the process. During ageing, specific compounds are transferred to wines as a result of prolonged contact with oak chips [30]. When employing the oak alternatives, the main factors to be considered are the amount and surface area (shape and size) of the materials used. In this respect, chip and barrel-aged wines differ in their content of a range of volatiles, such as vanillin, guaiacol or furfurals [3].

Louw et al. [44] states that young unoaked Chardonnay wines can be discriminated (with $74 \%$ correctly classified) by using the following eight volatile compounds: 2-phenylethanol, decanoic acid, diethyl succinate, ethyl hexanoate, ethyl decanoate, ethyl octanoate, hexyl acetate and 1-propanol. In general, alcohols can be the product of sugar fermentation and amino acid metabolism through yeasts. Higher alcohols have a significant impact on the fruity aroma of wine [45]. The major alcohols (iso-butanol, isopentyl alcohol and 2-phenylethanol) varied both depending on the time and type of ageing with different 
trends. However, at the end of the maturation period, the major alcohol concentrations were higher, regardless of the method. Also, the concentrations of the minor alcohols group were dependent on ageing time and method. The non-toasted barrel ageing method favored the concentration of hexanol, which supplied a leafy and grassy note to wine samples [31]. Generally, 2,3-butanediol is not expected to affect the sensory qualities of wine appreciably, but some authors [46] noted a bitter taste in variants with a concentration over $700 \mathrm{mg} / \mathrm{L}$ of this compound in wines samples. In our research, the higher concentration of 2,3 butanediol in the BAR variant wine sample could be explained by enzymatic reduction of diacetyl and acetoin in this compound under malolactic fermentation which could occur in barrels [47].

Overall, the time and method of ageing, significantly influenced the concentration of major and minor alcohols in wine samples, with higher amount for the variant aged in the non-toasted barrel. Increased amounts of higher alcohols during ageing can be caused by acid-catalyzed ester hydrolysis [48].

The esters chemical family contribute to the fruity character of wines and are one of the predominant chemical groups [49]. In this research, the impact of ageing duration and method on the Chardonnay wines concentration of esters was investigated. Most of them exhibited concentrations above their olfactory perception threshold, providing the wine with pear, apple, pineapple and/or floral notes [30,31]. During ageing, the esters had interesting trends; most of them have increased in concentration compared with the control wine and only hexyl acetate and trimethylene acetate decreased after ageing. Ethyl fatty acid esters are considered to be very important for the aroma of wines. The concentration of ethyl lactate progressively increases during malolactic fermentation [46]. This result is beneficial for the wine bouquet due to its fruity, buttery or creamy aromas, also contributing to the sensations of roundness in the mouth $[46,50]$. Overall, the ageing process, regardless of the method, slightly increased the amount of esters. Even more, after 1, 2 and 3 months of ageing, the concentrations of esters were nearly similar for the same time period of ageing among methods of ageing.

The fatty acids chemical group of volatile compounds is produced by yeast and bacteria during fatty acid metabolism. Volatile fatty acids can contribute to the complexity of the wine bouquet even if they are present under threshold levels. They have a negative effect on wine aroma when above their thresholds [51]. Some acids, such as iso-butyric acid and decanoic acid are not associated with wine quality, but they play an important role in the complexity of the aroma [52]. In the present study, there were significant differences in the fatty acid content between control wine and the wine samples aged by different methods. Decreased acid concentrations could be caused by the loss of volatile acids and esterification reactions between alcohols and acids during the ageing process [53]. Also, Vivas et al. [54] explained that the increase in volatile acidity in wine in contact with oak wood is mainly due to two reasons: firstly, the acidity may increase or decrease as a result of the chip roasting process, and secondly, may be due to acetic metabolism (i.e., acidic bacteria in wine).

As expected, the lactones showed the highest concentration in wine samples treated with non-toasted wood (non-toasted chips and barrel). This could be explained by the fact that some lactones are found in non-toasted wood, and thermal treatment degraded those compounds. Lactones add caramel and sweet flavor to wines [38].

Unlike the expected behavior, for ageing methods in/with non-toasted wood, total phenol volatiles content was similar for both methods, for the three ageing durations. It could be stated that, at least the first three months of ageing in non-toasted barrels are similar with ageing with non-toasted chips, regarding this chemical family. Regardless of the ageing method, wines which underwent the wood ageing presented greater vanillin concentrations, than the corresponding control wine. In our experiments, no control of MLF was conducted (no culture of bacteria and no inhibitors were added), so the increasing of vanilla volatile compounds in wood aged wine could under gone as the capacity of lactic bacteria (spontaneous MLF) to convert vanillin glycoside pre-cursors present in oak 
wood [55,56]. Spillman et al. [21] states that $p$-vinylguaiacol is a fermentation product rather than a compound derived from oak contact, formed through decarboxylation of natural grape components ferulic and $p$-coumaric acids by $S$. cerevisiae enzymatic activity. The concentration of 4-vinyl-guaiacol in our Chardonnay wine samples could indicate that this compound formed mainly during the fermentation period, but was also extracted from toasted wood products.

Overall woody aroma of barrel-aged wines results from different chemical compounds naturally present in oak wood, originated in the toasting process and/or formed as a consequence of the wood-wine interaction. Gambetta et al. [3] described furfurals, 5methylfurfural, cis- and trans-whiskey lactones, guaiacol, eugenol and vanillin as odorants derived from oak wood contact, or formed during ageing. The statistical test of the experimental data disclosed that the time and the ageing method significantly impact the oak wood volatiles concentration of the studied wines.

\subsection{Odor Activity Values}

Among all the compounds present in Table 3, not all of them present an odor activity value OAV $>1$ (Table 4 ), which is normally taken as the value required for a compound to be a likely contributor to the specific aroma. The OAVs based on threshold values in a specific matrix (e.g., neutral white wine or $10 \% v / v$ aqueous ethanol) act merely as a guide when determining the importance of a compound to wine aroma, due to a significant effect of the matrix [3]. The highest OAV was exhibited, among all samples, by ethyl octanoate characterized by pineapple and apple notes, specific for Chardonnay wines [19]. Ethyl hexanoate and $\mathrm{N}$-amyl acetate are fruity aroma contributors with concentrations above their threshold in all samples, mainly in wine samples treated with light-toasted chips. The method of wine ageing using non-toasted wood products, could preserve the floral character of Chardonnay wines. These results were in accordance with those presented previously by Calugar et al. [11], on Muscat Ottonel wines. Regarding the fatty acids, the rancid aroma supplied by isovaleric acid could be easily perceived in the non-toasted oak barrel aged sample, due to the low odor threshold of this compound. The spicy and condiment aroma could be highlighted by L1M-L3M samples, where light toast of chips increased the amount of $p$-vinyl guaiacol extracted by wine. The compounds that give wines a vanilla and roasted note (vanillin-VP5, $p$-vinyl guaiacol-VP1 and acetovanillone-VP3) are related to the light toasted chips in our study. For short periods of maturation, Chardonnay wines can obtain almost the same qualities if aged with nontoasted chips and non-toasted barrel, from the volatile point of view, but with more efficient cost, from the economical point of view. The PLRS analysis shows that wine samples treated with the French non-toasted oak chips aged for 1 or 2 months have similar wine volatile profiles with those from Romanian non-toasted oak barrels. Some differences between non-toasted variants were observed when the wines were aged for 3 months. Chardonnay is not dominated by a distinct varietal compound as in other varieties like Sauvignon blanc or Muscats. The typicity concept for Chardonnay wines needs more comprehension and requires deeper study, because this is a variety that can express itself in a multitude of profiles. Understanding Chardonnay typicity requires multidisciplinary studies that analyze a number of variables responsible for the final product, rather than a one-directional focus [3]. Nevertheless, the use of oak barrel alternatives, in particular, offers an economic means of imprinting the desirable aroma compounds from oak without the time or expenses associated with ageing wine in oak barrels [57].

It is not easy to distinguish products obtained by aging with chips from those obtained traditionally (barrel aged) from a sensory point of view. The distinction of the two different products is possible through laboratory investigation techniques: analytical methods allow expanding the compositional differences between the two wine products. Among the analytical techniques used, the spectroscopic ones are certainly those that, combined with multivariate statistics techniques, allow to obtain the best results in this field, for commercial purposes and, if necessary, for the control of fraudulent activities [58]. 


\section{Conclusions}

This work is the first study on volatile compounds of young and short matured Chardonnay wines from Romania. The method and time of ageing have a significant influence on basic wine parameters and on the volatile composition. The non-toasted medium (chips and barrel) ageing is correlated with lactones, esters (ethyl lactate and ethyl caproate) and fatty acids (isovaleric and lactic acids). The majority of volatile phenols were quantified in wines aged with light-toasted chips. The oak chip method of ageing the Chardonnay wines could be a useful tool to obtain wines as a viable alternative to the traditionally made Chardonnay wines. The amount of chips used in this study $(4 \mathrm{~g} / \mathrm{L})$ was selected in order to avoid an excessive impact of the wood character in wines that could produce a negative effect on tasting. For short periods of maturation, Chardonnay wines can obtain almost the same wine aged with non-toasted chips and non-toasted barrels, from the chemical point of view, but with more efficient cost, from the economic point of view. For white wines, the use of oak chips could avoid the oxidation of aromatic volatile compounds that could be produced during barrel ageing, and impart oak notes to wines without decreasing the fresh and fruity characteristics. This approach would enable diversification on the market and increase the range of products on offer to the consumer.

Author Contributions: Conceptualization, A.C., C.T., D.I.S. and E.G.; methodology, O.R.B., A.C.B. and A.B.; software, O.R.B.; validation, A.B., C.B., M.M. and A.M.; formal analysis, E.G. and A.C.; investigation, T.E.C.; resources, T.E.C., D.I.S. and C.T.; data curation, E.G.; writing-original draft preparation, A.C. and D.I.S.; writing-review and editing, T.E.C and C.T.; visualization, M.M.; supervision, T.E.C. and E.G.; project administration, D.I.S., A.C., T.E.C. and C.T. All authors have read and agreed to the published version of the manuscript.

Funding: This research received no external funding.

Institutional Review Board Statement: Not applicable.

Informed Consent Statement: Not applicable.

Data Availability Statement: Not applicable.

Acknowledgments: This work is supported by the project ANTREPRENORDOC, in the framework of Human Resources Development Operational Programme 2014-2020, financed from the European Social Fund under the contract number 36355/23.05.2019 HRD OP /380/6/13-SMIS Code: 123847.

Conflicts of Interest: The authors declare no conflict of interest.

\section{References}

1. Verdú, A.J.; Lloréns, F.; Fuentes, M. Measuring perceptions of quality in food products: The case of red wine. Food Qual. Pref. 2004, 15, 453-469. [CrossRef]

2. Charters, S.; Pettigrew, S. The dimensions of wine quality. Food Qual. Pref. 2007, 18, 997-1007. [CrossRef]

3. Gambetta, J.M.; Bastian, S.E.P.; Cozzolino, D.; Jeffery, D.W. Factors influencing the aroma composition of Chardonnay wines. J. Agric. Food Chem. 2014, 62, 6512-6534. [CrossRef] [PubMed]

4. Cejudo-Bastante, M.J.; Hermosin-Gutierrez, I.; Perez-Coello, M.S. Accelerated aging against conventional storage: Effects on the volatile composition of Chardonnay white wines. J. Food Sci. 2013, 78, C507-C5013. [CrossRef]

5. Martínez-Gil, A.; Del Alamo-Sanza, M.; Sánchez-Gómez, R.; Nevares, I. Different woods in cooperage for oenology: A Review. Beverages 2018, 4, 94. [CrossRef]

6. Cadahía, E.; Fernández de Simón, B.; Jalocha, J. Volatile compounds in spanish, french, and american oak woods after natural seasoning and toasting. J. Agric. Food Chem. 2003, 51, 5923-5932. [CrossRef] [PubMed]

7. Kyraleou, M.; Tzanakouli, E.; Kotseridis, Y.; Chira, K.; Ligas, I.; Kallithraka, S.; Teissedre, P.-L. Addition of wood chips in red wine during and after alcoholic fermentation: Differences in color parameters, phenolic content and volatile composition. OENO One 2016, 50. [CrossRef]

8. Herjavec, S.; Jeromel, A.; Orlic, S.; Kozina, B. Changes in chemical composition and sensory properties of Vugava wines aged in Croatia oak barrels. J. Cent. Eur. Agric. 2007, 8, 195-204.

9. Chira, K.; Teissedre, P.-L. Extraction of oak volatiles and ellagitannins compounds and sensory profile of wine aged with French winewoods subjected to different toasting methods: Behaviour during storage. Food Chem. 2013, 140, 168-177. [CrossRef] 
10. Gordillo, B.; Cejudo-Bastante, M.J.; Rodríguez-Pulido, F.J.; González-Miret, M.L.; Heredia, F.J. Application of the differential colorimetry and polyphenolic profile to the evaluation of the chromatic quality of Tempranillo red wines elaborated in warm climate. Influence of the presence of oak wood chips during fermentation. Food Chem. 2013, 141, 2184-2190. [CrossRef]

11. Tao, Y.; García Martín, J.F.; Sun, D.-W. Advances in wine aging technologies for enhancing wine quality and accelerating wine aging process. Crit. Rev. Food Sci. Nutr. 2014, 54, 817-835. [CrossRef]

12. Cortiella, M.; Ubeda, C.; Covarrubias, J.I.; Laurie, V.P.; Peña-Neira, Á. Chemical and physical implications of the use of alternative vessels to oak barrels during the production of white wines. Molecules 2021, 26, 554. [CrossRef] [PubMed]

13. Călugăr, A.; Coldea, T.E.; Pop, C.R.; Pop, T.I.; Babes, A.C.; Bunea, C.I.; Manolache, M.; Gal, E. Evaluation of volatile compounds during ageing with oak chips and oak barrel of muscat ottonel wine. Processes 2020, 8, 1000. [CrossRef]

14. Manolache, M.; Pop, T.I.; Babes, A.C.; Farcas, I.-A.; Muncaciu, M.L.; Călugăr, A.; Gal, E. Volatile composition of some red wines from romania assessed by GC-MS. Stud. UBB Chem. 2018, 63, 125-142. [CrossRef]

15. Chira, K.; Teissedre, P.-L. Relation between volatile composition, ellagitannin content and sensory perception of oak wood chips representing different toasting processes. Eur. Food Res. Technol. 2013, 236, 735-746. [CrossRef]

16. Commission Delegated Regulation (EU) 2019/934 of 12 March 2019 supplementing Regulation (EU) No 1308/2013 of the European Parliament and of the Council as regards wine-growing areas where the alcoholic strength may be increased, authorised oenological practices and restrictions applicable to the production and conservation of grapevine products, the minimum percentage of alcohol for by-products and their disposal, and publication of OIV files. Off. J. Eur. Union 2019, 62, 1-53.

17. Herrero, P.; Sáenz-Navajas, M.P.; Avizcuri, J.M.; Culleré, L.; Balda, P.; Antón, E.C.; Ferreira, V.; Escudero, A. Study of Chardonnay and Sauvignon blanc wines from D.O.Ca Rioja (Spain) aged in different French oak wood barrels: Chemical and aroma quality aspects. Food Res. Int. 2016, 89, 227-236. [CrossRef]

18. Guchu, E.; Díaz-Maroto, M.C.; Pérez-Coello, M.S.; González-Viñas, M.A.; Ibáñez, M.D.C. Volatile composition and sensory characteristics of Chardonnay wines treated with American and Hungarian oak chips. Food Chem. 2006, 99, 350-359. [CrossRef]

19. González-Centeno, M.R.; Chira, K.; Teissedre, P.L. Use of oak wood during malolactic fermentation and ageing: Impact on Chardonnay wine character. Food Chem. 2018, 278, 460-468. [CrossRef]

20. Liberatore, M.T.; Pati, S.; Del Nobile, M.A.; La Notte, E. Aroma quality improvement of Chardonnay white wine by fermentation and ageing in barrique on lees. Food Res. Int. 2010, 43, 996-1002. [CrossRef]

21. Spillman, P.J.; Sefton, M.A.; Gawel, R. The contribution of volatile compounds derived during oak barrel maturation to the aroma of a Chardonnay and Cabernet Sauvignon wine. Austr. J. Grape Wine Res. 2004, 10, 227-235. [CrossRef]

22. Antoce, A.O.; Călugăru, L.L. Evolution of grapevine surfaces in Romania after accession to European Union-period 2007-2016. In Proceedings of the 40th World Congress of Vine and Wine, BIO Web of Conferences, Sofia, Bulgaria, 29 May-2 June 2017; Volume 9, pp. 03018.

23. Irimia, L.M.; Patriche, C.V.; Rosca, B.; Cotea, V.V. Modifications in climate suitability for wine production of Romanian wine regions as a result of climate change. In Proceedings of the 40th World Congress of Vine and Wine, BIO Web of Conferences, Sofia, Bulgaria, 29 May-2 June 2017; Volume 9, pp. 01026. [CrossRef]

24. OIV. Compendium of International Methods of Wine and Must Analysis Paris, France, 2020. Available online: http://www.oiv. int/public/medias/7372/oiv-compendium-volume-1-2020.pdf (accessed on 18 September 2020).

25. Bora, F.D.; Donici, A.; Oşlobanu, A.; Fițiu, A.; Babes, A.C.; Bunea, C.I. Qualitative assessment of the white wine varieties grown in Dealu Bujorului vineyard, Romania. Not. Bot. Horti Agrobot. 2016, 44, 593-602. [CrossRef]

26. Andujar-Ortiz, I.; Moreno-Arribas, M.V.; Martín-Álvarez, P.J.; Pozo-Bayón, M.A. Analytical performance of three commonly used extraction methods for the gas chromatography-mass spectrometry analysis of wine volatile compounds. Adv. Sep. Methods Food Anal. 2009, 1216, 7351-7357. [CrossRef] [PubMed]

27. Ivanova, V.; Stefova, M.; Vojnoski, B.; Stafilov, T.; Bíró, I.; Bufa, A.; Felinger, A.; Kilár, F. Volatile composition of macedonian and hungarian wines assessed by GC/MS. Food Bioprocess Technol. 2013, 6, 1609-1617. [CrossRef]

28. Etiévant, X.P. Wine. In Volatile Compounds in Foods and Beverages; Maarse, H., Ed.; Marcel Dekker: New York, NY, USA, 1991; pp. 583-546.

29. Pomar, M.; Gonzalez-Mendoza, L.A. Changes in composition and sensory quality of red wine aged in American and French oak barrels. J. Int. Sci. Vigne Vin. 2001, 35, 41-48. [CrossRef]

30. Dumitriu, G.-D.; Teodosiu, C.; Gabur, I.; Cotea, V.V.; Peinado, R.A.; López de Lerma, N. Evaluation of aroma compounds in the process of wine ageing with oak chips. Foods 2019, 8, 662. [CrossRef]

31. Cheng, G.; Liu, Y.; Yue, T.-X.; Zhang, Z.-W. Comparison between aroma compounds in wines from four Vitis vinifera grape varieties grown in different shoot positions. Food Sci. Technol. Campinas 2015, 35, 237-246. [CrossRef]

32. Baron, M.; Prusova, B.; Tomaskova, L.; Kumsta, M.; Sochor, J. Terpene content of wine from the aromatic grape variety 'Irsai Oliver' (Vitis vinifera L.) depends on maceration time. Open Life Sci. 2017, 12, 42-50. [CrossRef]

33. Guth, H. Quantification and sensory studies of character impact odorants of different white wine varieties. J. Agric. Food Chem. 1997, 45, 3027-3032. [CrossRef]

34. Ferreira, V.; Lopez, R.; Cacho, J.F. Quantitative determination of the odorants of young red wines from different grape varieties. J. Sci. Food Agric. 2000, 80, 1659-1667. [CrossRef]

35. Du, X.F.; Finn, C.E.; Qian, M.C. Volatile composition and odour-activity value of thornless 'Black Diamond' and 'Marion' blackberries. Food Chem. 2010, 119, 1127-1134. [CrossRef] 
36. Furtuna, N. Harnessing the Aroma Potential of Grape Varieties Startovyi, Viorica and Muscat of Ialoveni. Ph.D. Thesis, Tehnic University of Moldova, Chișinau, Moldova, 2015; 169p.

37. Návojská, J.; Brandes, W.; Nauer, S.; Eder, R.; Frančáková, H. Influence of different oak chips on aroma compounds in wine. J. Microbiol. Biotechnol. Food Sci. 2012, 1, 957-971.

38. Farrell, R.R.; Wellinger, M.; Gloess, A.N.; Nichols, D.S.; Breadmore, M.C.; Shellie, R.A.; Yeretzian, C. Real-time mass spectrometry monitoring of oak wood toasting: Elucidating aroma development relevant to oak-aged wine quality. Sci. Rep. 2015, 5, 17334. [CrossRef]

39. Aiken, J.W.; Noble, A.C. Composition and sensory properties of Cabernet Sauvignon wine aged in French versus American oak barrels. Vitis 1984, 23, 27-36.

40. Towey, J.P.; Waterhouse, A.L. The extraction of volatile compounds from French and American oak barrels in Chardonnay during three successive vintages. Am. J. Enol. Vitic. 1996, 47, 163-172.

41. Garde-Cerdán, T.; Torrea-Goñi, D.; Ancín-Azpilicueta, C. Accumulation of volatile compounds during ageing of two red wines with different composition. J. Food Eng. 2004, 65, 349-356. [CrossRef]

42. Garde-Cerdán, T.; Lorenzo, C.; Carot, J.M.; Jabaloyes, J.M.; Esteve, M.D.; Salinas, M.R. Statistical differentiation of wines of different geographic origin and aged in barrel according to some volatile components and ethylphenols. Food Chem. 2008, 111, 1025-1031. [CrossRef]

43. Ortega-Heras, M.; González-Sanjosé, M.L.; González-Huerta, C. Consideration of the influence of aging process, type of wine and oenological classic parameters on the levels of wood volatile compounds present in red wines. Food Chem. 2007, 103, 1434-1448. [CrossRef]

44. Louw, L.; Tredoux, A.G.J.; Van Rensburg, P.; Kidd, M.; Naes, T.; Nieuwoudt, H.H. Fermentation-derived aroma compounds in varietal young wines from South Africa. S. Afr. J. Enol. Vitic. 2010, 31, 213-225. [CrossRef]

45. Cameleyre, M.; Lytra, G.; Tempere, S.; Barbe, J.-C. Olfactory impact of higher alcohols on red wine fruity ester aroma expression in model solution. J. Agric. Food Chem. 2015, 63, 9777-9788. [CrossRef]

46. Lasik-Kurdys, M.; Majcher, M.; Nowak, J. Effects of different techniques of malolactic, fermentation induction on diacetyl metabolism and biosynthesis of selected aromatic esters in cool-climate grape wines. Molecules 2018, 23, 2549. [CrossRef]

47. Martineau, B.; Henick-Kling, T. Performance and diacetyl production of commercial strains of malolactic bacteria in wine. J. Appl. Bacteriol. 1995, 78, 526-536. [CrossRef]

48. Rapp, A.; Mandery, H. Wine aroma. Experientia 1986, 42, 873-884. [CrossRef]

49. Welke, J.E.; Zanus, M.; Lazzarotto, M.; Alcaraz, Z.C. Quantitative analysis of headspace volatile compounds using comprehensive two-dimensional gas chromatography and their contribution to the aroma of Chardonnay wine. Food Res. Int. 2014, 59, 85-99. [CrossRef]

50. Ugliano, M.; Moio, L. Changes in the concentration of yeast-derived volatile compounds of red wine during malolactic fermentation with four commercial starter cultures of Oenococcus Oeni. J. Agric. Food Chem. 2005, 53, 10134-10139. [CrossRef] [PubMed]

51. Swiegers, J.H.; Pretorius, I.S. Yeast modulation of wine flavour. Adv. Appl. Microbiol. 2005, 57, 131-175.

52. Shinohara, T. Gas chromatographic analysis of volatile fatty acids in wines. Agr. Biol. Chem. Tokio 1985, 49, 2211-2212.

53. Xu, M.L.; Yu, Y.; Ramaswamy, H.S.; Zhu, S.M. Characterization of chinese liquor aroma components during aging process and liquor age discrimination using gas chromatography combined with multivariable statistics. Sci. Rep. 2017, 7, 39671-39671. [CrossRef] [PubMed]

54. Vivas, N.; Lonvaud Funel, A.; Glories, Y. Observations concerning the increase of volatile acidity in red wines whilst ageing in barrels. J. Sci. Tech. Tonnelerie Fr. 1995, 1, 81-122.

55. Bloem, A.; Lonvaud-Funel, A.; de Revel, G. Hydrolysis of glycosidically bound flavour compounds from oak wood by Oenococcus oeni. Food Microbiol. 2008, 25, 99-104. [CrossRef]

56. Martínez-Gil, A.M.; Garde-Cerdán, T.; Martínez, L.; Alonso, G.L.; Salinas, M.R. Effect of oak extract, application to Verdejo grapevines on grape and wine aroma. J. Agric. Food Chem. 2011, 59, 3253-3263. [CrossRef] [PubMed]

57. Arapitsas, P.; Antonopoulos, A.; Stefanou, E.; Dourtoglou, V.G. Artificial aging of wines using oak chips. Food Chem. 2003, 86, 563-570. [CrossRef]

58. Petrozziello, M.; Nardi, T.; Asproudi, A.; Cravero, M.C.; Bonello, F. Chemistry and technology of wine aging with oak chips chemistry and biochemistry of winemaking. Cosme, F., Nunes, F.M., Filipe-Ribeiro, L., Eds.; Intech Open: London, UK, 2020. [CrossRef] 